\title{
Welcome to SIGNALS: A New Open-Access Scientific Journal on Signal Analysis, Retrieval and Processing
}

\author{
Andrzej Cichocki 1,2,3 \\ 1 Skolkovo Institute of Science and Technology (SKOLTECH), 143026 Moscow, Russia; a.cichocki@riken.jp \\ 2 Systems Research Institute, Polish Academy of Science, 01-447 Warsaw, Poland \\ 3 College of Computer Science, Hangzhou Dianzi University, 310018 Hangzhou, China
}

Received: 13 June 2018; Accepted: 14 June 2018; Published: 19 June 2018

The sheer exposure to vast amounts of signals created in our modern society and an ever increasing need to make sense of such data calls for continuing advances in signal processing as an enabling technology for a huge number of applications, ranging from wireless communication and medicine, through to bioengineering, the economy and entertainment. Despite enormous diversity in physical and physiological signal generating mechanisms in such applications, the mathematical background at the core of the majority of signal detection, estimation, classification or fusion algorithms is common to most practical applications, both current and future. It is important to note that we consider the notion of signals in a general sense, to include not only signals processed and manipulated by computers or smart devices but also by means of biological systems such as our brains. Such biological systems process information from sensors in an intelligent way to perform even the most complex of tasks such as recognizing objects, sounds, and taste or smooth navigation in complex and cluttered environments.

The goal of this journal is therefore to answer the need to communicate the large volume of exciting research activity in this area, by providing a new platform for academicians, scientists and engineers to share, promote, and discuss various new issues and developments in the areas concerned with signals, images and videos. The aim of the MDPI International Journal SIGNALS is to promote and expedite the dissemination, not only of new research results, but also of tutorial and review-type articles focusing on current trends and the state-of-the-art on all subjects relevant to analysis and/or processing of signals in either discrete or continuous time and their applications, especially intelligent/smart signal/data processing. The signals of interest can include sound and audio, time-varying measurements and sensor data, biological data such as electroencephalogram (EEG), neuroimages, including functional magnetic resonance images (fMRI) and positron emission tomography (PET), control system signals, telecommunication transmission signals, and more abstract signals like multidimensional time series or tensors.

There are a variety of scientific journals devoted to signal processing, and the SIGNALS journal is developing its own profile with a special focus on intelligent signal processing, blind signal processing, blind source separation, and smart biomedical/bioengineering applications. In recent years, artificial intelligence (AI) and machine learning (ML) have become well-established research areas and attract a large number of researchers across the fields of science and engineering, and include deep learning, big data, IoT, and smart things. Recent dramatic improvements in artificial intelligence and machine learning have only reinforced the importance of signal and image processing research, especially in biomedical signal processing. Our goal in this Journal is therefore to enhance and promote these trends towards Intelligent Signal/Data Processing.

The editorial policy of SIGNALS is to publish high quality research papers which contain substantial mathematical contributions and also validations/evaluation of performance by extensive computer simulations. Mathematically and machine learning oriented papers on topics of exceptional interest to the signal processing community will also be considered. Priority will be given to 
contributions concerned with a discussion of the background of practical problems, establishment of appropriate models, determination of a solution, approximate or exact, analytical or numerical, and a discussion of the relevance of the results when applied to the real-life problems.

Papers are solicited from, but not limited to the following topics:

- Activity recognition, event detection, anomaly detection

- Adaptive filtering and signal processing

- Applications of signal processing (Biomedical, Bioinformatics, Genomic, Seismic, Radar, Sonar, Remote Sensing, Positioning, Embedded Systems, etc.)

- Array signal processing

- Audio/speech processing and coding

- Biometrics and authentification

- Biomedical Signal Processing and biomedical data analysis (EEG/MEG, fMRI, PET etc.)

- Biological network and data analysis/modelling

- Biosignal processing and understanding

- Blind and semi-blind signal separation (e.g., single- and multi-channel recordings, audio source separation, bio-signal separation, independent component analysis (ICA), nonnegative matrix/tensor factorization (NMF/NTF)

- Brain Computer Interface (BCI)

- Communication signal processing

- Compressive Sensing (CS) and sparse information retrieval

- Computer Vision (CV) and Virtual Reality (VR)

- Computational Neuroscience and brain data analysis/modelling

- Cryptography and network security

- Emerging technologies

- Image processing and understanding

- Image/Video Processing and Coding

- Inference and prediction of hidden patterns in signals/images

- Multimedia signal processing

- Natural Language Processing (NLP)

- Neuroimaging and signal processing

- Signal processing for human-computer interaction (HCI) and Brain Computer Interface (BCI).

Furthermore, the journal has a special emphasis on Tensor Signal Processing and Statistical Signal Processing methodology such as Bayesian signal processing, and encourages articles from emerging areas in signal processing which do not find space in other journals of the field. These include:

- Image and multidimensional signal processing using tensor representation

- Scalable and distributed algorithms for tensor processing

- Methods for robust tensor processing with noisy, incomplete and/or missing signals/data

- Tensor methods in neural networks and deep learning

- Tensor-based time series and image processing

- Tensor processing and analysis in social networks and economics

- Applications of tensor processing in wireless communications and sensor networks

- Tensor developments in computer vision

- Tensor methods in neuroscience and medicine

and

- Stochastic modelling of biological processes, systems biology, 
- Information theory articles with a signal processing perspective

- Deep learning probabilistic neural networks with signals/data processing perspective

- Financial time series analysis

- Bioinformatics and neuroinfomatics

- Biochemical interaction in biological and biologically-inspired systems

- Signal processing for performance arts using audio, and video

- $\quad$ Signal processing for renewable energy

- Game theory and group theory for signal processing

- Environmental signal processing

- Signal processing with intelligent user interfaces

- Quantum signal processing.

Signals analysis and signal processing will continue to play a key role in the development of new technologies and improving the quality of our daily life, especially in smart and intelligent systems, to extract, recognize, detect, classify, extract transmit useful information. We hope that the MDPI open access journal SIGNALS will serve and facilitate these new exciting developments. 